\title{
Emergency Door Capacity: Influence of Door Width, Population Composition and Stress Level
}

\author{
W. Daamen* and S. P. Hoogendoorn, Faculty of Civil Engineering \\ and Geosciences, Department of Transport \& Planning, Delft University \\ of Technology, PO Box 5048, 2600 GA Delft, The Netherlands
}

Received: 23 February 2010/Accepted: 18 November 2010/Published online: 16 December 2010

\begin{abstract}
For the new version of the Dutch design guidelines for buildings, a threshold value for the capacity of emergency doors needs to be set. Innovative largescale laboratory experiments have been performed to investigate the capacity of emergency doors during evacuation conditions. This paper focuses in particular on the relation between capacity and the independent variables doorway width, population composition, stress level and presence of an open door. It turned out that only the experiment with the widest doorway $(275 \mathrm{~cm})$ resulted in a capacity lower than the capacity from the current design guidelines $(2.25 \mathrm{P} / \mathrm{m} / \mathrm{s})$. The average observed capacities are for all widths lowest for the lowest stress level and highest for the highest stress level. The population with a greater part of children has the highest capacity (on average $3.31 \mathrm{P} / \mathrm{m} / \mathrm{s}$ ). This is mainly due to the smaller physical size of children compared to adults and elderly, which makes it possible that more children can pass a door at the same time than adults. The lowest capacity (on average $2.02 \mathrm{P} / \mathrm{m} / \mathrm{s}$ ) is found for the experiment with $5 \%$ disabled participants. The presence of a door opened in the escape direction in an angle of $90^{\circ}$ for a doorway of $85 \mathrm{~cm}$ results in a $20 \%$ capacity reduction compared to the reference experiment. The open door does not physically narrow the doorway, but it leads to interactions between participants reducing their speed and the corresponding outflow.
\end{abstract}

Keywords: Capacity of emergency doors, Population, Door width, Laboratory experiments, Stress level

\section{Introduction}

Since 1992, the Dutch national building code ("Building decree") sets requirements to the width of emergency doors. Since 2003, these requirements depend on the number of persons that rely on an emergency door. According to the Building decree a door width of $1 \mathrm{~m}$ is sufficient to let 135 persons pass during the period available for safe escape (1 min). This value corresponds to research of Peschl [1], being similar to $2.25 \mathrm{P} / \mathrm{m} / \mathrm{s}$.

The threshold of 135 persons per meter width during a safe escape time of 1 min has been discussed for years between the Ministry for Housing, Regional

\footnotetext{
* Correspondence should be addressed to: W. Daamen, E-mail: w.daamen@tudelft.nl
} 
Development and the Environment and the fire brigades that are used to allow a maximum of 90 persons per meter width during a safe escape time of $1 \mathrm{~min}$ $(1.5 \mathrm{P} / \mathrm{m} / \mathrm{s})$.

A literature research has been performed to find other research related to similar bottlenecks. In 2002, the department Transport and Planning of the Delft University of Technology performed laboratory experiments with a narrow bottleneck. This resulted in a capacity of $1.77 \mathrm{P} / \mathrm{m} / \mathrm{s}$ [2], which is closer to the capacity recommended by the fire brigades than in the design guidelines. However, the narrow bottleneck spans a short hallway of five meters long, whereas a doorway usually has a length of $10-40 \mathrm{~cm}$. This will most likely lead to a higher capacity for doorways, since pedestrians may accept short headways for a short period of time.

Kretz et al. [3] performed bottleneck experiments as well. In these experiments, the bottleneck was a thick wall of $40 \mathrm{~cm}$ with an opening the pedestrians had to pass. Different widths for the opening have been considered $(40 \mathrm{~cm}, 50 \mathrm{~cm}, 60 \mathrm{~cm}$, $70 \mathrm{~cm}, 80 \mathrm{~cm}, 90 \mathrm{~cm}, 100 \mathrm{~cm}, 120 \mathrm{~cm}, 140 \mathrm{~cm}$, and $160 \mathrm{~cm}$ ). The participants consisted of healthy students; the experimental conditions were normal. A linear decrease of the capacity is shown with increasing bottleneck width as long as only one person at a time can pass (from $2.2 \mathrm{P} / \mathrm{m} / \mathrm{s}$ for $40 \mathrm{~cm}$ to $1.78 \mathrm{P} / \mathrm{m} / \mathrm{s}$ for $70 \mathrm{~cm}$ width). A constant value of the capacity of around $1.8 \mathrm{P} / \mathrm{m} / \mathrm{s}$ is shown for larger bottleneck widths $(70 \mathrm{~cm}, 80 \mathrm{~cm}, 100 \mathrm{~cm}$ and $120 \mathrm{~cm})$. Only the very narrow bottleneck thus shows a capacity slightly lower than the capacity indicated in the design guidelines, the other bottlenecks width result in lower capacities.

Experimental research by Müller [4] and Nagai et al. [5] indicated much higher capacities for bottleneck widths varying between $80 \mathrm{~cm}$ and $160 \mathrm{~cm}$, namely between $2.29 \mathrm{P} / \mathrm{m} / \mathrm{s}$ and $3.23 \mathrm{P} / \mathrm{m} / \mathrm{s}$. These high values can be explained by the very high densities at the start of the experiments. Also, the configuration of the bottleneck is slightly different, which affects the measured capacities [6]. These values correspond to the threshold indicated in the design guidelines.

Many observations have been performed in corridors and in areas with many pedestrians present (e.g., stations, inner cities, and stadiums), for overviews see [7, 8]. The capacities found vary between $1.03 \mathrm{P} / \mathrm{m} / \mathrm{s}$ and $1.67 \mathrm{P} / \mathrm{m} / \mathrm{s}$, thus much lower than the design guidelines. However, these capacities are found in normal conditions, which most likely will lead lower capacities than in evacuation conditions.

The abovementioned overview indicates a wide variety of capacities found, while most of the capacities are lower than the capacities in the design guidelines. The aim of the research described in this paper is therefore to perform experimental research to collect new information on the capacity of doors during emergency conditions.

The next section describes the set up of the experiments in more detail. It also gives a short impression of the day of the experiments. In the third section the methodology is described to calculate the capacities, the results of which are shown in the next section. We end with conclusions and recommendations for future research. 


\section{Experimental Set Up}

The capacity of an emergency door depends on several aspects, among which the composition of the population using the door, the conditions under which the door is used and the door width. Before describing these experimental variables in more detail, some boundary conditions are set.

In the experiments, an opening represents the emergency door: subjects pass a free passage of a certain width. In this opening, no doorstep is present, to reduce hindrance and prevent possibly dangerous situations for participants. In addition, the pedestrian flow is one-directional, implying that no counter flows are present caused by fire fighters and people from emergency services. In reality, these people will rarely enter a building when the evacuation process is still going on.

The experiments performed by Peschl [1] have been based on a student population. However, in practice, the population will not consist of persons being in good shape, but the persons will have different physical conditions. In this research, we will use age as an indication for a person's physical condition. Here, we distinguish four categories: children (under 18 years of age), adults (between 18 and 65 years of age), elderly (over 65 years of age) and disabled persons. With these age categories, we are able to compose populations corresponding to a variety of situations, see Table 1 . The disabled people are represented by three persons in wheelchairs and three blindfolded persons.

The conditions under which an emergency door is used may vary considerably. In the experiments, both the stress level of the participants and the sight are varied. Not much is known on how to introduce stress in an experiment. In the past two methods have been considered favorable: enforcing participants to hurry e.g., by rewarding participants according to their performance and exposing participants to noise. Here, we have chosen to use for the latter option by sounding the slow-whoop signal. In addition, the stress level of the participants is raised by a combination of the slow-whoop signal and stroboscope light. In total, participants have been exposed to three stress levels: none, a slow-whoop signal and a combination of a slow-whoop signal and stroboscope light.

The sight is reduced by reducing illumination to a low level. Two alternative light situations are considered: full lighting (200 lux) and dimmed (1 lux, corresponding to emergency lighting).

\section{Table 1}

\section{Overview of Different Populations in the Experiments}

\begin{tabular}{llcccc}
\hline \multicolumn{1}{c}{ Population } & Children (\%) & Adults (\%) & Elderly (\%) & Disabled (\%) \\
\hline 1 & School & 90 & 10 & 0 & 0 \\
2 & Station during peak hours & 0 & 100 & 0 & 0 \\
3 & Retirement home & 5 & 20 & 75 & 0 \\
4 & Work meeting & 5 & 90 & 5 & 0 \\
5 & Shopping centre & 30 & 60 & 10 & 0 \\
6 & Average & 25 & 55 & 20 & 0 \\
7 & Disabled & 23 & 54 & 18 & 5 \\
\hline
\end{tabular}


In the experiments, the opening width is varied between $50 \mathrm{~cm}$ (the minimal free passageway of an escape route in the Building decree for existing buildings) and $275 \mathrm{~cm}$. In addition to an opening of $85 \mathrm{~cm}$ wide (minimal free passageway of an escape route in the Building decree for new estates) openings are a multiple of $55 \mathrm{~cm}$. Furthermore, an opening of $100 \mathrm{~cm}$ is tested to see the correspondence with the normative capacity expressed as the number of persons passing an opening of $1 \mathrm{~m}$ wide in $1 \mathrm{~min}$.

The final experimental variable relates to whether or not the outflow of pedestrians after passing the doorway is free. In reality, doors cannot always open $180^{\circ}$, but may be restricted. To restrict the outflow of pedestrians after passing the doorway, we used a door being fixed at an opening of $90^{\circ}$. The total doorway width is not affected.

Ideally, all combinations of experimental variables should be investigated. Since this is not feasible due to time restrictions (the experiments should not last longer than a single day), for each experiment one variable is changed, while for the other variables the default value is maintained. By interpolation of the results of the various experiments, pronouncements can be made on the not performed experiments. The stress levels are varied for all experiments.

Each experiment will be performed multiple times to guarantee the reliability of the observations. To determine the number of repetitions, a total time of congestion of $3 \mathrm{~min}$ should be achieved. Since the time of congestion for wide doors is shorter than for narrow doors, more repetitions are performed for the wide doors.

An overview of the experiments is shown in Table 2.

A digital video camera and an infrared camera are used to observe the experiments. The infrared camera observes LEDs, attached on top of the caps of the

\section{Table 2}

\section{Overview of the Performed Experiments}

\begin{tabular}{lclcccc}
\hline Experiment & Opening width $(\mathrm{cm})$ & Population & Sight (lux) & Open door & Start time & Repetitions \\
\hline 1 & 100 & Average & 200 & No & $9: 58$ & 12 \\
2 & 220 & Average & 200 & No & $10: 17$ & 22 \\
3 & 85 & Retirement home & 200 & No & $10: 43$ & 12 \\
4 & 85 & Average & 200 & No & $10: 58$ & 10 \\
5 & 165 & Average & 1 & No & $11: 25$ & 22 \\
6 & 275 & Average & 200 & No & $11: 52$ & 23 \\
7 & 85 & Work meeting & 200 & No & $12: 49$ & 12 \\
8 & 85 & Disabled & 200 & No & $12: 23$ & 12 \\
9 & 85 & School & 200 & No & $13: 48$ & 12 \\
10 & 85 & Average & 1 & No & $14: 08$ & 9 \\
11 & 50 & Average & 200 & No & $14: 24$ & 6 \\
12 & 110 & Average & 200 & No & $14: 39$ & 15 \\
13 & 85 & Shopping centre & 200 & No & $15: 19$ & 12 \\
14 & 85 & Average & 200 & Yes & $15: 40$ & 9 \\
15 & 165 & Average & 200 & No & $16: 03$ & 16 \\
16 & 85 & Station & 200 & No & $16: 24$ & 11 \\
\hline
\end{tabular}

\footnotetext{
${ }^{a}$ Slightly different average composition with relatively fewer children
} 


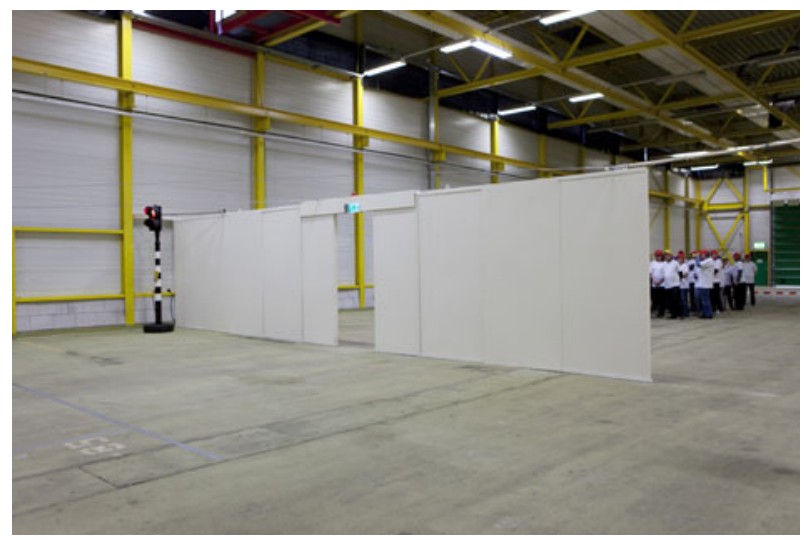

\section{Fig. 1. Overview of the experimental site.}

participants. This technique guarantees good observations for the dimmed conditions. For the other experiments a digital camera is used, which is attached to the ceiling next to the infrared camera.

In total 75 children of 11 years old (blue caps), 90 adults (red caps) and 50 elderly persons (yellow caps) have participated in the experiments. This leads to populations of between 90 and 150 persons, which are large enough to cause congestion upstream of the door to observe capacities.

To represent an emergency door, a wall has been built in the middle of a large hallway, perpendicular to the sidewall. In this wall, an opening is made, whose width is easy to vary. At the side of the wall, some space is left to walk from one side of the wall to the other without using the opening. Above the centre of the opening an emergency exit sign has been hung up. An overview of the experimental site is shown in Fig. 1. To use the doorway more efficiently the participants use it in two directions: in the first experiment, they walk from one side of the wall to the other and in the next experiment they walk back again.

\section{Methodology to Calculate Emergency Door Capacity}

The images of the digital video camera form the basis to calculate the capacity of an emergency door. The movie of each repetition of an experiment is split into separate images with a frequency of 25 images per minute. Figure 2 a shows such an image of the reference experiment with a doorway of $85 \mathrm{~cm}$ wide, an average population, 200 lux, no open door and no stress.

To calculate the capacity of the emergency door, the moments that the subjects pass a cross-section directly downstream of the door need to be known. Figure $2 b$ shows the considered cross-sections: the dotted line indicates the cross-section to determine the capacity of the flow from right to left, while the striped line corresponds to the cross-section to determine the capacity of the flow from left to right.

To determine the cumulative curves on the specified cross-sections, the picture lines of the cross-section are placed next to each other for the duration of a repe- 


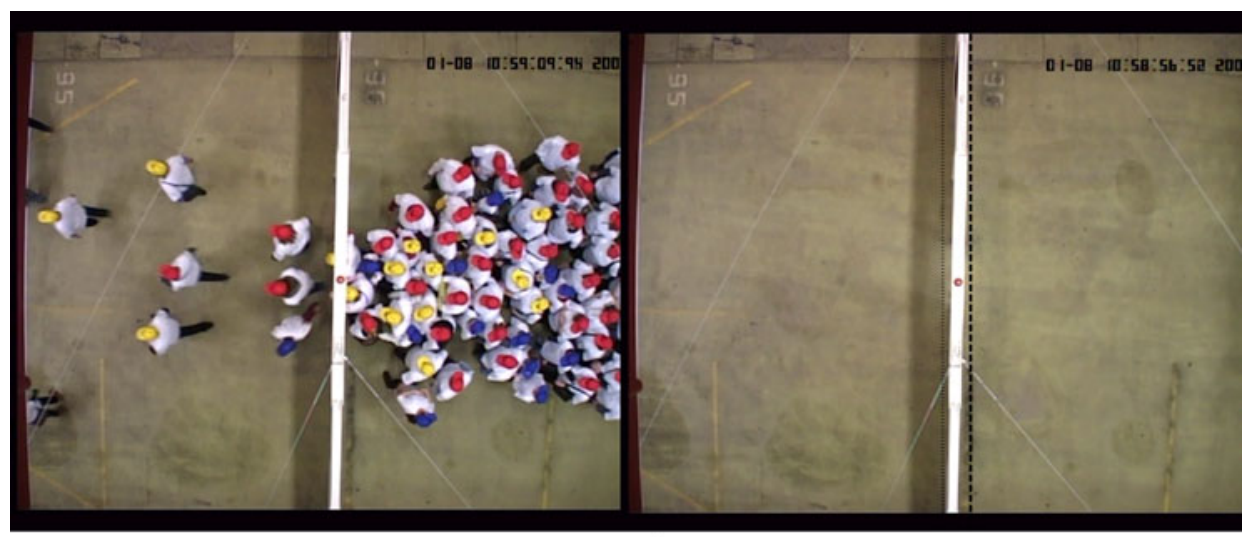

$\mathbf{a}$

b

\section{Fig. 2. Digital video camera images of the reference experiment. (a) Overview of the pedestrians, (b) cross sections to measure capacity.}

tition of an experiment using MATLAB ${ }^{\circledR}$. This gives a similar effect as a finish photo, see Fig. 3a. Figure $3 \mathrm{~b}$ shows a zoom of the picture lines in Fig. 3a. This figure clearly shows that the first participants have passed the cross-section at high speed: their image is very narrow, while the images of the following persons are wider. A similar approach has been used to identify vehicle trajectories from video images made from a helicopter [9].

The next step is to recognize the caps to automatically derive the passage moments of the individuals using the cap colors. In the 'photo finish' image areas are looked for within a pre-specified color range. By not only looking for the colors bright red, bright blue and bright yellow, but by allowing a color range, the shadow effects, the cap edges and the stroboscope effects are compensated for. The detected pixels are then combined to caps. For this, two built-in methods in Matlab $^{\circledR}$ have been used: a method to identify areas within a specific color range and clustering methods. More information on this cap recognition methodology can be found in [10]. Figure $3 \mathrm{c}$ shows the tracked participants for a repetition of the reference experiment.

For each cluster (participant) its heart is calculated. The x-pixel of this heart indicates the time moment when the participant passes the cross-section, while the y-pixel indicates the lateral position of this event. A cumulative curve can be derived based on these passage moments, where the number of persons having passed the cross-section is plot against the passing moment, see Fig. $3 \mathrm{~d}$.

Assuming that the capacity of the door does not change during a repetition of the experiment, a straight line is fit through the cumulative curve. The derivative of this line corresponds to the average capacity of this door during this repetition. The average capacity of the experiment is then the average of the capacities of all repetitions. If the average capacity of each experiment is known, the relations between the capacity and the various experimental variables (door width, population, stress level, etc.) can be determined. 

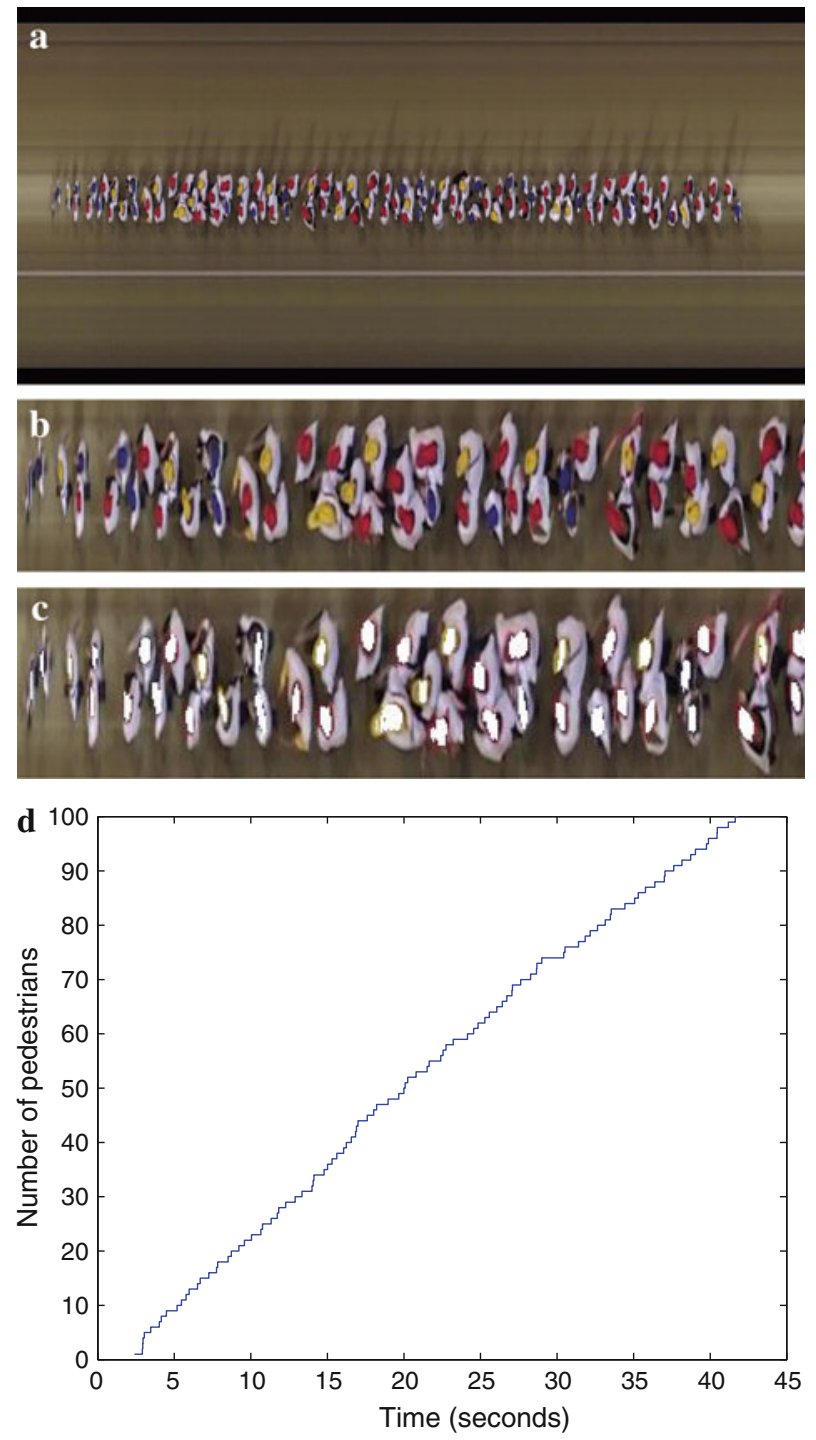

Fig. 3. Picture lines (a), zoom of the picture lines (b), and overview of the tracked pedestrians (c) of a repetition of the reference experiment and the resulting cumulative curve (d).

\section{Relations Between Capacities and Experimental Variables}

Based on the methodology described in the previous section capacities have been calculated for all repetitions of all experiments. In this section, the influence of the experimental variables, doorway width, population composition and the influence of an open door are discussed. 


\subsection{Doorway Width}

During the experiments the opening width has been varied between $50 \mathrm{~cm}$ and $275 \mathrm{~cm}$. All these experiments have been performed with an average population (with a slight change for the widest doorway), a normal light intensity (200 lux) and without the presence of an open door. Figure 4 shows the results of these experiments.

For each experiment, the observed capacity is shown in the figure. The type of marker indicates the stress level, while the green star represents the average capacity per experiment over all stress level. In addition, the current threshold capacity from the Building decree has been indicated $(\mathrm{C}=2.25 \mathrm{P} / \mathrm{m} / \mathrm{s}=135 \mathrm{P} / \mathrm{min})$.
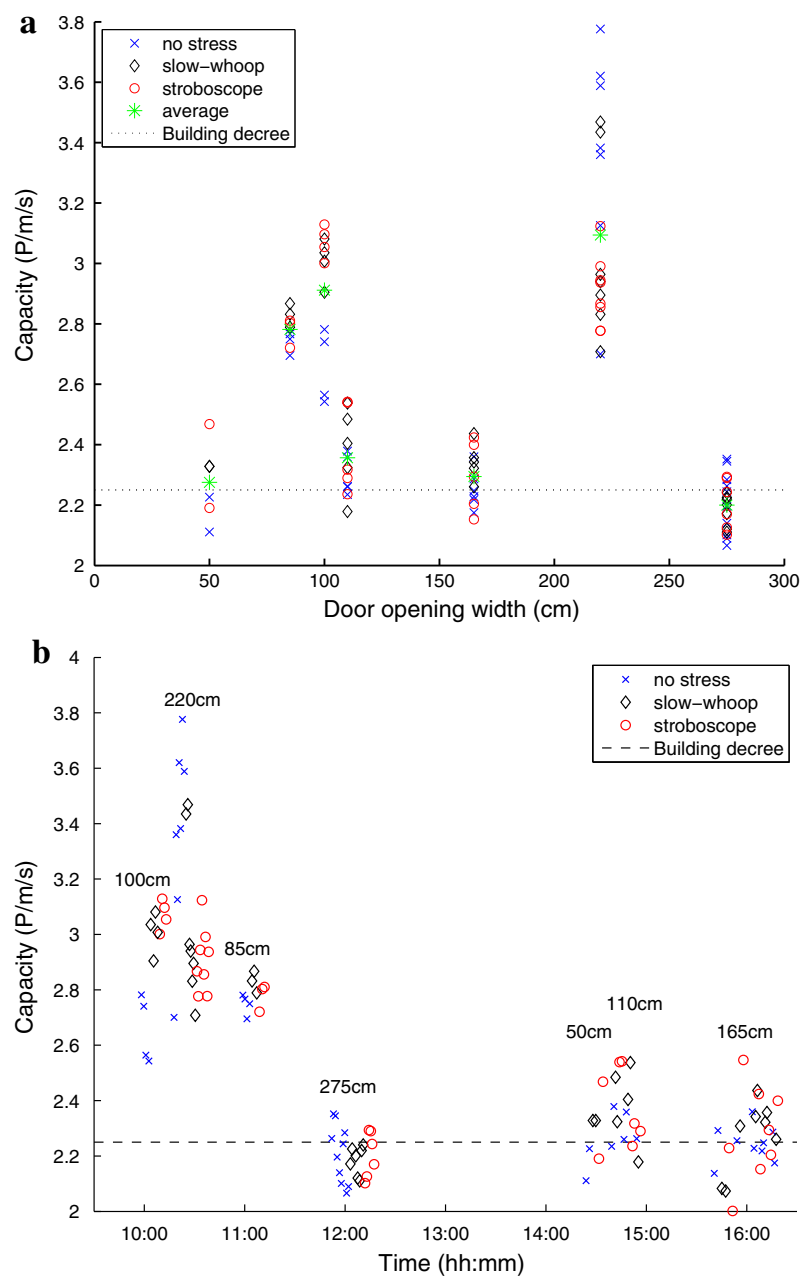

Fig. 4. Capacity as a function of doorway width (a) and time of day (b) for an average population, a light intensity of 200 lux and no open door present. 
The figure shows that only the experiment with the widest doorway results in an average capacity lower than the threshold value from the Building decree. Furthermore, the high capacity of the doorway of $220 \mathrm{~cm}$ is remarkable, as well as the large difference between the repetitions in this experiment. Figure 4 indicates that the repetitions without stress or with a low stress level result in the highest capacity for a doorway of $220 \mathrm{~cm}$, while the experiment for a doorway of $100 \mathrm{~cm}$ contradicts this finding. Figure 5 gives more insight into the influence of the various stress levels.

Figure 5 shows that the average observed capacities over all doorway widths are lowest for the lowest stress level and highest for the experiments with slowwhoop and stroboscope considered as the highest stress level. For all cases the average observed capacities are much higher than the value included in the Building decree. As well as in Fig. 4a, the figure shows some outliers for the experiments without stress and with only a slow-whoop signal for a doorway of $220 \mathrm{~cm}$. An explanation can be found in the time of the day this experiment has taken place (see Fig. 4b).

Figure $4 \mathrm{~b}$ indicates that both experiments with the largest variance in capacity have taken place at the beginning of the morning. Moreover, the figure shows that the stress level has an opposite effect for both experiments: for the first experiment the capacity is lowest for the lowest stress level, while in the second experiment the highest capacities occur at the lowest stress level. The other experiments do
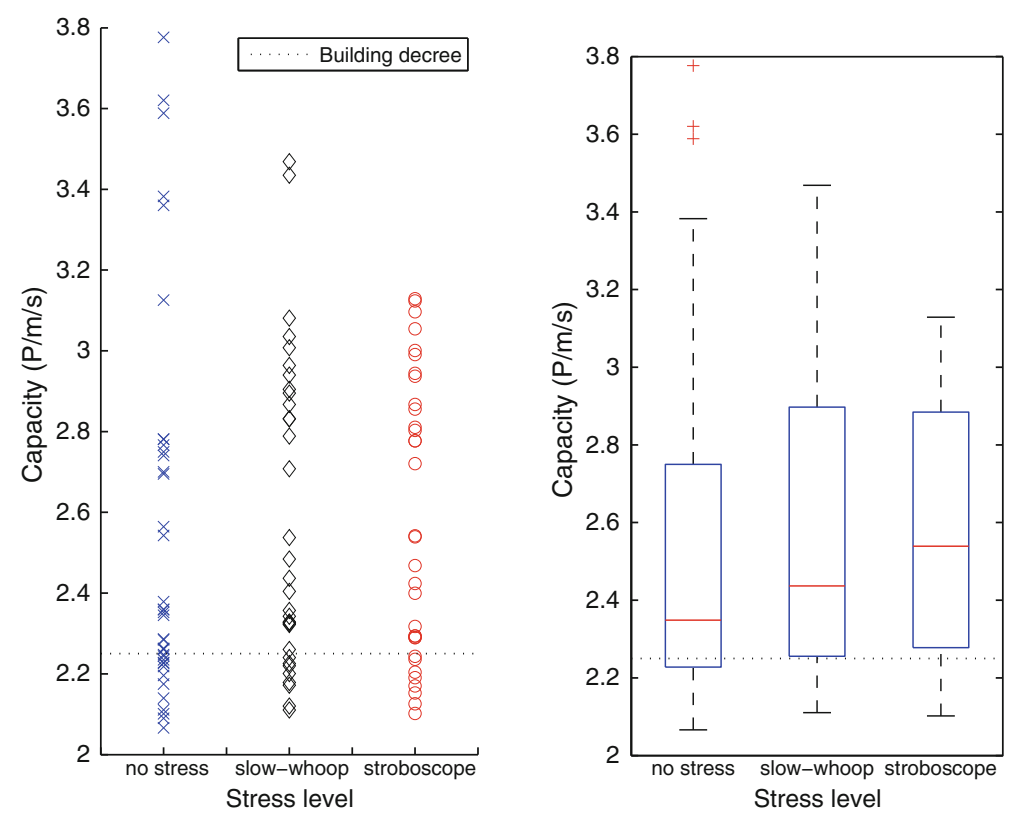

Fig. 5. Capacity as function of stress level for the various doorway widths for an average population, a light intensity of 200 lux and no open door present. On the left the individual observations are shown, on the right a box plot. 
not show such a clear effect of the various stress level. This leads to the conclusion that the difference is not structural and can be attributed to the conditions (enthusiasm) during the first experiments. Although the number and the distribution of the participants over the three groups (children, adults, elderly) are equal for all experiments, other persons participated. While the participants of the first experiment did not know what to deal with, the participants of the second experiment could wait and see what was happening. Especially at the start of the experiment, these participants were very motivated and were in full focus to pass the door. In the first repetitions (without stress and with slow-whoop, respectively) this led to pushy behavior; this was clearly visible in the video images.

Although the behavior of the participants appears to influence the capacity, it is impossible to motivate the participants just as much for each experiment during the total day. Despite this fact, the capacity of almost all repetitions is higher than the capacity prescribed in the Building decree. Only most repetitions of the experiment with the widest opening are below the capacity threshold from the Building decree. Since the first experiments showed that the capacities appeared to be higher than the planned capacities all adults and elderly have joined this experiment. This led to a slightly different population with relatively less children than the average population, which has a negative effect on the capacity as will be shown in the following. This slightly adapted average population only participated in the experiment with the widest door; in all other experiments the regular average population has participated.

\subsection{Population Composition}

During the experiments also the population has been varied. These experiments have been performed with a doorway of $85 \mathrm{~cm}$ wide, a normal light intensity (200 lux) and without an open door. Figure 6 shows the results of these experiments.

The figure above shows that five out of six experiments result in a capacity higher than the capacity threshold indicated in the Building decree. Only the population with $5 \%$ disabled persons (three blindfolded participants and three participants in wheelchairs) results in a slightly lower capacity $(2.0 \mathrm{P} / \mathrm{m} / \mathrm{s}$ vs. $2.25 \mathrm{P} / \mathrm{m} / \mathrm{s})$. The population with mainly children has the highest capacity. This is mainly caused by the physical fact that children are smaller than adults, which makes it possible for more children to pass a door at the same time. The populations representing a retirement home, a meeting and a shopping centre do not differ much. Conversely, the capacity of the population 'station' varies considerably from the population 'meeting'. The first population consists only of adults, while the second population consists of $90 \%$ adults, completed with $5 \%$ children and $5 \%$ elderly. However, the difference between both capacities is somewhat more than $8 \%$. Also the population 'shopping centre' and 'average' have a substantially different capacity $(15 \%)$, while the first population has only $5 \%$ more children, $5 \%$ more adults and $10 \%$ less adults. These differences might be explained by the moment of the day the experiment has been performed (see Fig. 6b).

For both situations mentioned above the performance moment of the experiment has a clear but opposite effect. The experiment with the average population 

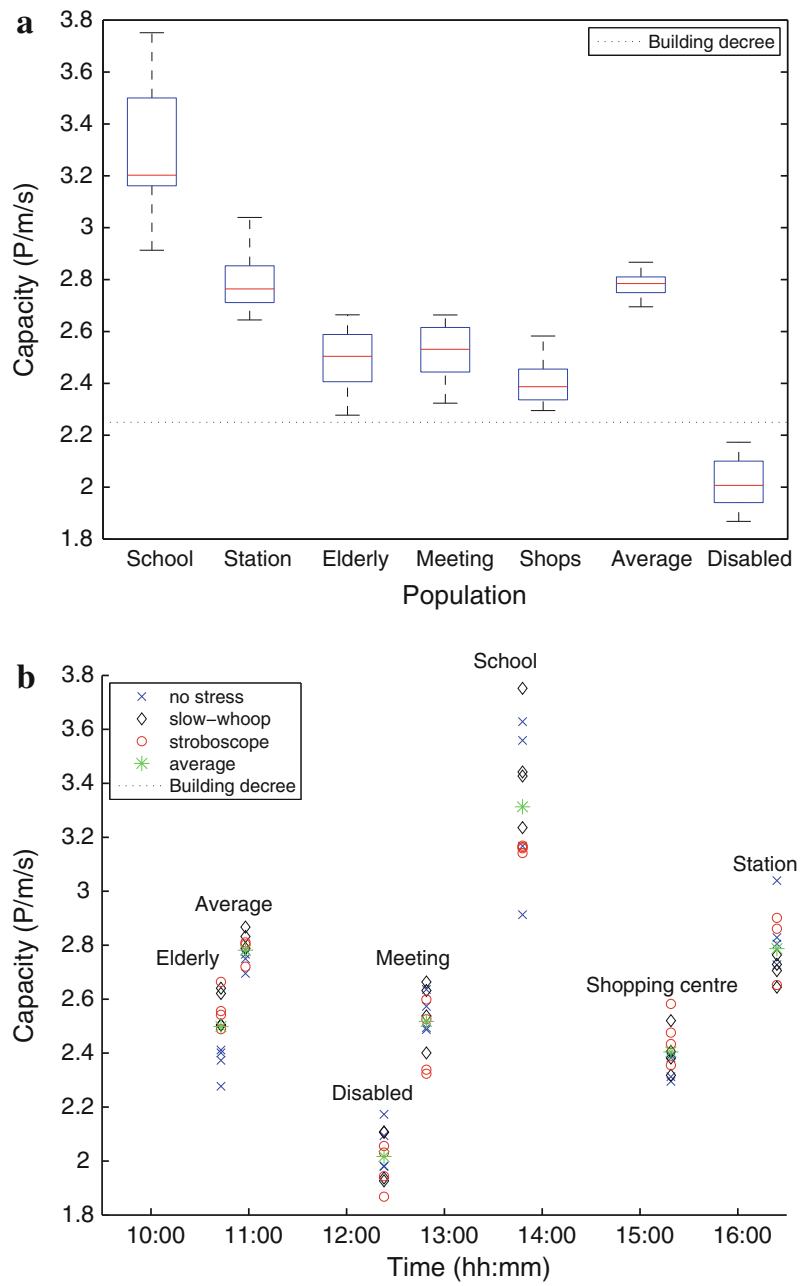

\section{Fig. 6. Capacily as function of population (a) and time of day (b) at a doorway of $85 \mathrm{~cm}$, a light intensity of 200 lux and no open door.}

was the fourth experiment of the day just before a short break, while the experiment with the shopping centre population occurred halfway the afternoon. At that moment the fatigue had increased considerably and the enthusiasm decreased, which lead to a lower capacity than the capacity of a comparable average population. Exactly the opposite causes the difference in capacity between the meeting population and the station population. The experiment with the meeting population occurred by one o'clock, when the participants were clearly in need of a lunch break, while the experiment with the station population occurred at the end of the day. To motivate the participants of the station population (consisting of only adults) extra, the challenge was raised to improve the highest capacity until then, set by the school population ("let's see if you can evacuate faster than the 
children"). This lead to a very strong motivation, resulting in a much higher capacity than the one of a similar population.

The variation in capacity is highest for the school population, which can be attributed to the fact that children strongly react to each other: if the first person passes the doorway very fast, the others will follow very fast as well, whereas if the first person passes the doorway very slow, the others will also take it easy. However, the variation between the experiments with the stroboscope was very small, probably because this unusual external condition makes the children focus more on the aim of the experiments (less distraction).

\subsection{Presence of an Open Door}

The last experimental variable discussed in this paper is the presence of an open door. Figure 7 shows the results for this experimental variable.

From the figure it can be concluded that the capacity decreases up to $80 \%$ of the capacity threshold indicated in the Building decree when an open door is present in the doorway. This door does not physically narrow the doorway, but it reduces the outflow of the participants. At the location of the door some kind of narrow corridor exists, while on the other side participants are only hindered in their lateral movement at the moment of passing the wall. In the situation without a door participants fan out in all directions immediately after passing the door, which is not possible when a door is present. This is clearly visible in the
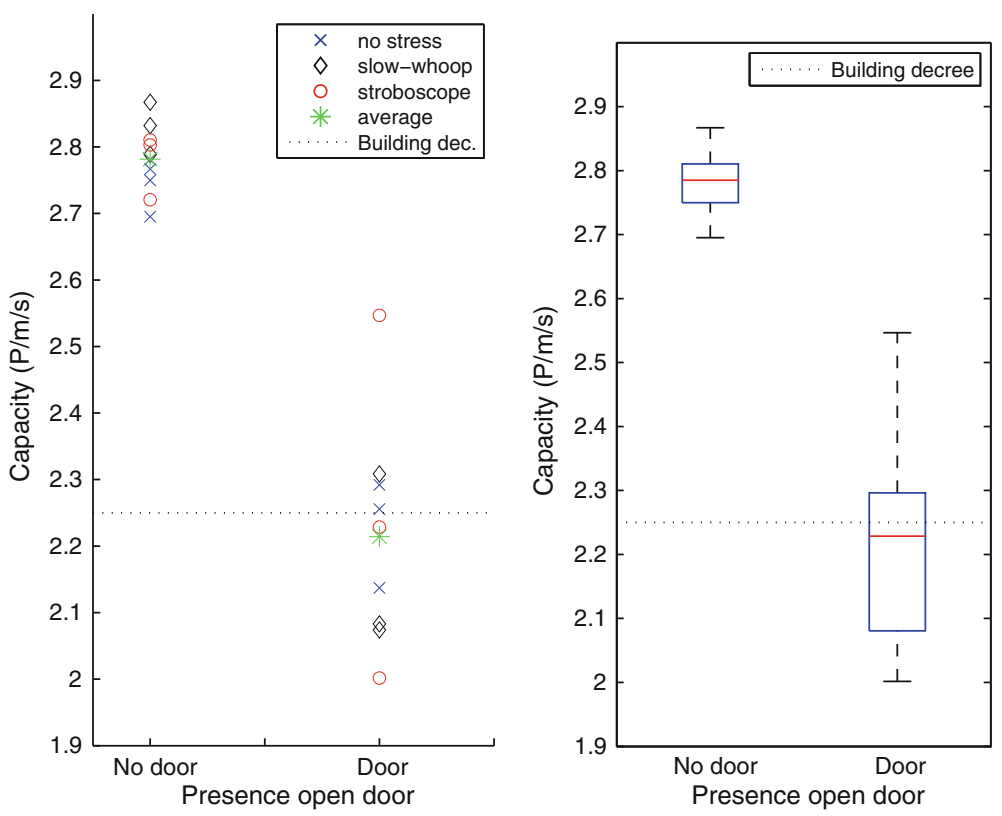

Fig. 7. Capacity as a function of the presence of an open door for a doorway of $85 \mathrm{~cm}$, an average population and a light intensity of 200 lux. 

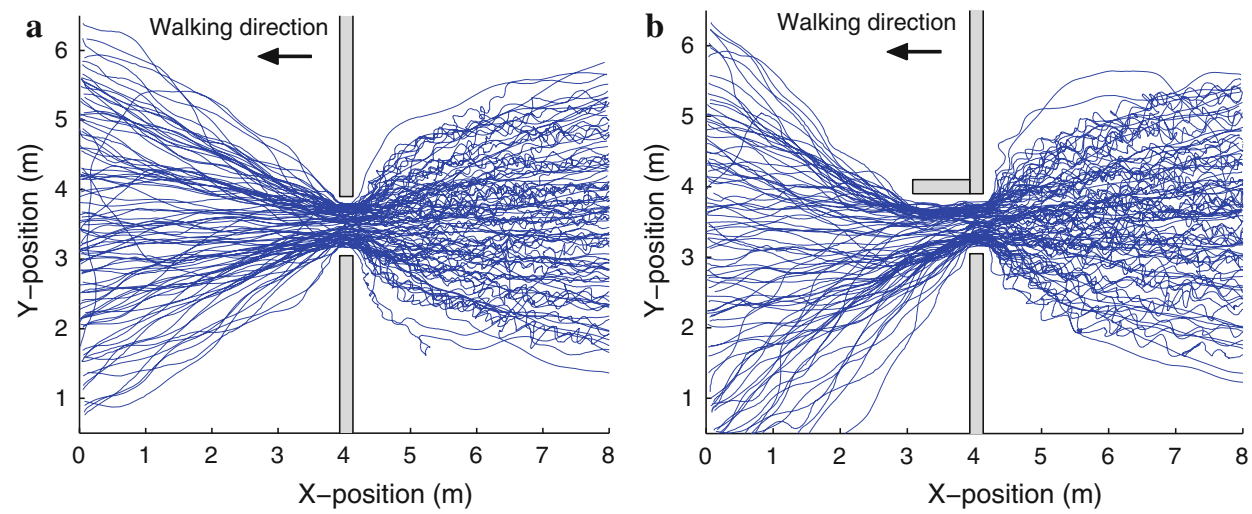

\section{Fig. 8. Trajectories for a doorway of $85 \mathrm{~cm}$ wide without (a) and with (b) open door.}

trajectories for both situations (see Fig. 8) and an overview of the use of the area downstream of the door (see Fig. 9, the darker the areas, the more frequently this area is used).

When an open door is present, not only less surface directly downstream of the doorway is used, but this surface is also used more intensely (darker areas in Fig. 9). The fact that the doorway is not fully used is not caused by pedestrians maintaining distance to the door, but because pedestrians cannot directly swerve to the right downwards of the wall. This is slightly compensated by pedestrians passing the opening at the left hand side swerving more to the left to give space to pedestrians passing the opening at the right hand side. The angle between the used surface on the left hand side of the wall and the wall is therefore smaller than in the situation that no door is present. Figure 9 also shows that a door not necessarily has to open $180^{\circ}$, since the surface directly behind the wall is not used. A maximum opening angle of $150^{\circ}$ appears to be sufficient for a free outflow.

The intense use of the surface immediately downstream of the door results in many interactions between pedestrians, which leads to lower speeds and thus to a lower outflow (and thus capacity) at the doorway.

\section{Multivariate Regression}

In the previous section, the influence of each independent variable on the capacity has been discussed separately. In this section we will show the influence of all variables together, performing a multivariate regression. Here, we take into account stress-level $S(0=$ no stress; $1=$ slow whoop signal; $2=$ slow whoop signal combined with stroboscope), door width $W$ in meter, population (percentage of children $P_{C}$, percentage of elderly $P_{E}$ and percentage of disabled persons $\left.P_{D}\right)$, the presence of an open door $D(0 / 1)$, light intensity $L(0.05 / 1)$ and time $T$ in hours from the starting moment. For the population, we have left out the 

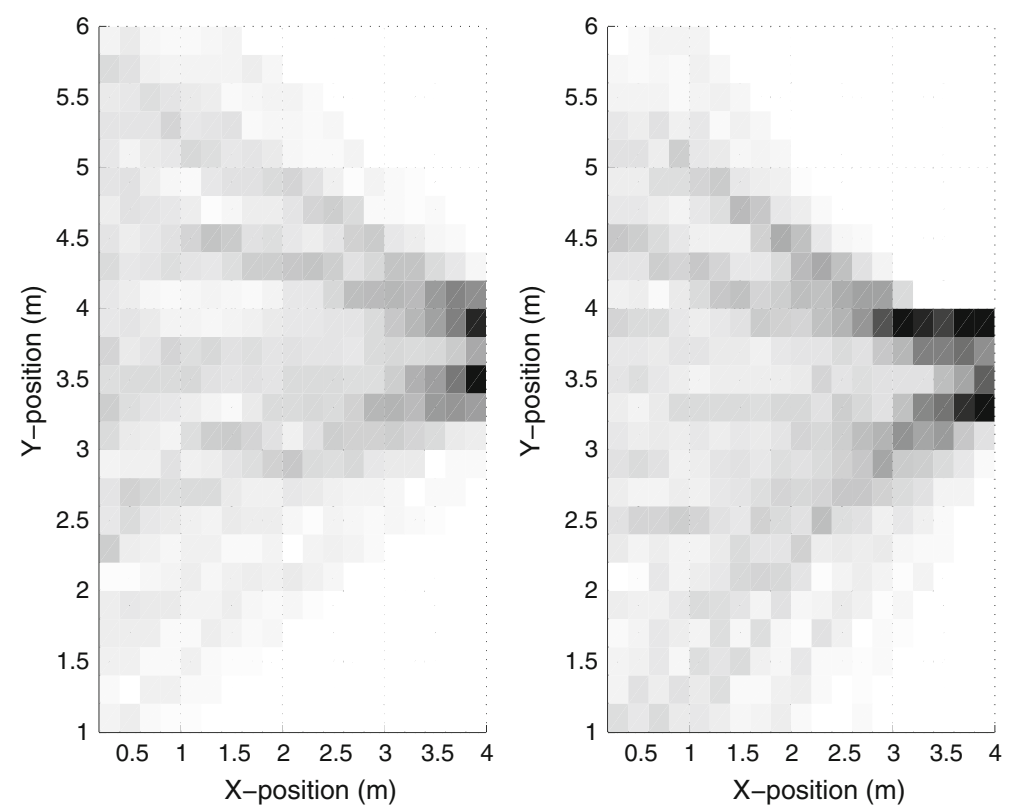

Fig. 9. Surface use after passing the doorway without open door (left) and with open door (right). In both figures the opening is sifuated between $y=3.1 \mathrm{~m}$ and $\mathrm{y}=\mathbf{3 . 9 5} \mathrm{m}$ at the lateral position of $x=1.7 \mathrm{~m}$.

percentage of adults $P_{A}$, since this is redundant with the other percentages $\left(P_{A}=1-P_{C}-P_{E}-P_{D}\right)$. The capacity $C$ can then be calculated as follows:

$$
\begin{aligned}
C= & 2.6685-0.0065 S-0.1153 W+1.0612 P_{C}-0.2077 P_{E} \\
& -2.1310 P_{D}-0.1789 D+0.0895 L-0.0850 T
\end{aligned}
$$

Equation 1 shows that the reference capacity is $2.6685 \mathrm{P} / \mathrm{m} / \mathrm{s}$. The stress level hardly has an effect on the capacity. The door width also has a negative effect, implying that wider doors are less efficiently used. Obviously, the door width for which Eq. 1 can be applied is limited, otherwise the capacity becomes negative. Since the widest door handled in the experiments had a width of $2.75 \mathrm{~m}$, we suggest to take $3 \mathrm{~m}$ as boundary width. With respect to the population, we can see that the children have the largest, positive, effect on capacity: the more children are part of the population, the higher the capacity. A higher percentage of elderly also has a slightly positive effect, whereas more disabled persons in the population have a strong negative effect on the capacity. The presence of an open door also has a negative effect on the capacity, whereas the lighting conditions have a positive effect (more light indicates a higher capacity). Finally, as we saw in earlier analyses, the time moment that the experiment took place has a slightly negative effect on capacity. 
Table 3

T-Test for the Significance of the Parameters $(95 \%$ Significant if Test Value >1.644854)

\begin{tabular}{lccrc}
\hline Variable & Value & Standard error & Test value & Significant \\
\hline Stress level & 0.0065 & 0.0165 & 0.3943 & No \\
Door width & 0.1153 & 0.0139 & 8.2998 & Yes \\
\% Children & 1.2690 & 0.0668 & 15.8955 & Yes \\
\% Elderly & 0.2077 & 0.0837 & 2.4811 & Yes \\
\% Disabled & 1.9233 & 1.2782 & 1.6672 & Yes \\
Open door & 0.1789 & 0.1022 & 1.7508 & Yes \\
Light & 0.0895 & 0.0226 & 3.9616 & Yes \\
Time & 0.0850 & 0.0058 & 14.6132 & Yes \\
\hline
\end{tabular}

\section{Table 4}

\section{Correlation Between Variables}

\begin{tabular}{lrrrrrrrrr}
\hline & Stress & Width & Children & Elderly & Disabled & Open door & Light & Time & Constant \\
\hline Stress & 0.0006 & 0.0000 & -0.0000 & 0.0000 & 0.0004 & -0.0000 & -0.0000 & 0.0000 & -0.0007 \\
Width & 0.0000 & 0.0012 & 0.0006 & 0.0010 & 0.0107 & 0.0003 & -0.0001 & 0.0001 & -0.0023 \\
Children & -0.0000 & 0.0006 & 0.0204 & 0.0118 & 0.0325 & 0.0010 & -0.0006 & -0.0000 & -0.0081 \\
Elderly & 0.0000 & 0.0010 & 0.0118 & 0.0294 & 0.0198 & -0.0006 & -0.0008 & 0.0006 & -0.0116 \\
Disabled & 0.0004 & 0.0107 & 0.0325 & 0.0198 & 2.0522 & 0.0275 & -0.0118 & -0.0028 & -0.0213 \\
Open dr & -0.0000 & 0.0003 & 0.0010 & -0.0006 & 0.0275 & 0.0118 & -0.0005 & -0.0003 & 0.0002 \\
Light & -0.0000 & -0.0001 & -0.0006 & -0.0008 & -0.0118 & -0.0005 & 0.0036 & -0.0001 & -0.0023 \\
Time & 0.0000 & 0.0001 & -0.0000 & 0.0006 & -0.0028 & -0.0003 & -0.0001 & 0.0001 & -0.0006 \\
Constant & -0.0007 & -0.0023 & -0.0081 & -0.0116 & -0.0213 & 0.0002 & -0.0023 & -0.0006 & 0.0126 \\
\hline
\end{tabular}

The next question is whether these parameters are significant. For this, we applied the $t$-test for each parameter. Table 3 shows that only the parameter for the stress level is not significant.

Finally, we checked the correlation between the parameters. Table 4 shows that the variables are not correlated.

\section{Conclusions and Recommendations}

The main conclusion that can be drawn is that the capacity of thirteen out of sixteen experiments is higher than $2.25 \mathrm{P} / \mathrm{m} / \mathrm{s}$. The experiments with a lower capacity have a population with disabled persons, a very wide doorway $(275 \mathrm{~cm})$ with less children in the population and an open door.

Another conclusion is that in the performed experiments more pushing does not lead to the 'faster-is-slower' effect. In the experiments a higher urgency (higher stress level) leads to higher speeds and to a higher capacity.

During the experiments, the conditions were optimal. This implies among other things that the inflow of pedestrians towards the door is unhindered, which is also the case for the outflow (except for the experiment with the presence of an open 
door). Also, factors such as people wearing winter clothing or carrying bags have not been taken into account. In order to determine the design value of the capacity of an evacuation door, users of the building code and designers of buildings should realize themselves in which conditions their doors will be used, and adapt the capacity value accordingly.

Many differences between the observed capacities can be explained by the different experimental variables. The images of the experiments indicate that an explanation can also be found in the individual behavior of the participants. When this microscopic behavior can be predicted, also the capacities can be predicted for a larger variety of conditions. This will be subject of future research.

The research described here has explicitly been focused on the capacity of emergency doors. This is only part of the total evacuation process. The previous process (pre-evacuation, route choice, walking towards the exit) has a direct influence on the arrival pattern of pedestrians at the emergency door, and thus whether or not capacity of the door will be reached. This is also subject of future research.

\section{Acknowledgment}

The research presented in this paper is part of the research program "Predictable accessibility of buildings", sponsored by the Dutch Foundation of Scientific Research MaGW-NWO. The authors are grateful to the anonymous reviewers for their valuable comments that helped improving the paper.

\section{Open Access}

This article is distributed under the terms of the Creative Commons Attribution Noncommercial License which permits any noncommercial use, distribution, and reproduction in any medium, provided the original author(s) and source are credited.

\section{References}

1. Peschl IASZ (1971) Evacuation capacity of door openings in panic situations. Bouw 26:62-67in Dutch

2. Hoogendoorn SP, Daamen W (2005) Pedestrian behavior at bottlenecks. Transp Sci 39(2):147-159

3. Kretz T, Grünebohm A, Schreckenberg M (2006) Experimental study of pedestrian flow through a bottleneck. J Stat Mech P10014:1-23

4. Müller K (1981) The design and measurement of routes for evacuation of persons from building. Dissertation, Technische Hochschule Magdeburg, Magdeburg (in German)

5. Nagai R, Fukamachi V, Nagatani T (2006) Evacuation of crawlers and walkers from corridor through an exit. Phys A 367:449-460

6. Seyfried A, Passon O, Steffen B, Boltes M, Rupprecht T, Klingsch W (2009) New Insights into pedestrian flow through bottlenecks. Transp Sci Arctic Adv. doi: $10.1287 / \operatorname{trsc} .1090 .0263$ 
7. Daamen W (2004) Modelling passenger flows at public transport facilities. PhD Dissertation, Delft University of Technology, The Netherlands

8. Buchmüller S, Weidmann U (2006) Parameters of pedestrians, pedestrian traffic and walking facilities. IVT-Report 132, Institute for Transport Planning and Systems (IVT), Swiss Federal Institute of Technology Zurich (ETHZ)

9. Knoop VL, Hoogendoorn SP, Van Zuylen HJ (2009) Processing traffic data collected by remote sensing. Transp Res Rec: J Transp Res Board 2129:55-61

10. Hoogendoorn SP, Daamen W (2002) Extracting microscopic pedestrian characteristics from video data. In: Proceedings of the annual meeting of the transportation research board. CD-ROM. Transportation Research Board, National Research Council, Washington, DC 